\title{
Le paradoxe de la rébellion : les relations inter- raciales aux Etats Unis
}

\section{Dipak Gupta}

\section{(2) OpenEdition}

\section{Journals}

Édition électronique

URL : http://journals.openedition.org/conflits/437

DOI : $10.4000 /$ conflits.437

ISSN : $1777-5345$

Éditeur :

CCLS - Centre d'études sur les conflits lilberté et sécurité, L'Harmattan

Édition imprimée

Date de publication : 15 décembre 1993

ISSN : 1157-996X

Référence électronique

Dipak Gupta, «Le paradoxe de la rébellion : les relations inter-raciales aux Etats Unis », Cultures \&

Conflits [En ligne], 12 I hiver 1993, mis en ligne le 14 mars 2006, consulté le 30 mars 2021. URL : http:// journals.openedition.org/conflits/437 ; DOl : https://doi.org/10.4000/conflits.437

Ce document a été généré automatiquement le 30 mars 2021.

Creative Commons License 


\section{Le paradoxe de la rébellion : les relations inter-raciales aux Etats Unis}

Dipak Gupta

Les images télévisées des émeutes de Los Angeles ont ouvert, pour le reste du monde, une fenêtre sur l'état des relations inter-raciales aux Etats-Unis. Ajoutées aux diffusions répétées de reportages sur la montée du néo-nazisme et des mouvements de la Suprématie Blanche, les scènes d'anomie de Los Angeles peuvent apparaître, pour de nombreux téléspectateurs (et surtout ceux des pays étrangers) comme la manifestation d'une violente lutte ethnique. Au regard du monde, où les hostilités ouvertes entre groupes ethniques semblent plus être la règle que l'exception, il est légitime de se poser la question de l'avenir des relations inter-raciales aux Etats-Unis. Sont-elles destinées à emprunter les chemins du conflit politique violent comme celui d'Irlande du nord, de l'ex-Yougoslavie, des anciennes Républiques de l'ex-Union Soviétique ou encore comme ceux existant dans de nombreux autres pays d'Afrique ou d'Asie? Bien qu'il soit difficile de répondre à cette vaste question, l'objet de cet article est de resituer les émeutes de Los Angeles dans une perspective particulière et de tenter de comprendre pourquoi la communauté afro-américaine n'a pas réussi à traduire sa frustration et sa colère dans une forme de lutte politique cohérente alors même qu'elle est confrontée à un accroissement de son niveau de "frustration" économique et à une dépossession répétée de ses droits politiques. Les émeutes de 1992 remettent en mémoire l'été 1967, période particulièrement sombre pour la nation américaine. Les émeutes du début des années soixante avaient brutalement remis en cause le mythe du melting-pot. La perception insidieuse d'une marmite dont le couvercle s'ouvre brusquement sous l'action de l'accumulation de la vapeur sous pression remplaçait l'image de ce meltingpot. Le Président Johnson soulignait, aux vues des circonstances, l'urgence de la situation en prévenant qu'il s'agissait là d'un signal d'alarme et que la nation devait prendre des mesures pour apaiser la situation avant qu'il ne soit trop tard. La Commission Kerner, mise en place en 1967 afin d'étudier les causes de la révolte urbaine rendait son rapport un an plus tard ${ }^{1}$. Dans ce rapport la Commission parvenait, 
ce n'est pas une surprise, aux même conclusions. La Commission Kerner attribuait la responsabilité des grandes émeutes urbaines et des mécontentements qui régnaient parmi les citoyens noirs du pays à la quasi ségrégation économique présente dans la société. Citant pour exemples la disparité des réalisations dans le domaine des salaires, de la santé, du logement et de l'éducation, la Commission évoquait, à moins bien sûr que la nation ne prenne en considération la nécessité de combler l'inégalité raciale, la probabilité de l'imminence d'un vaste affrontement. Ainsi, la Commission estimait: Notre Nation se dirige vers l'établissement de deux sociétés, une noire, une blanche séparées et inégales. ${ }^{2}$ La réaction aux désordres de l'été dernier a accéléré le mouvement et accentué la division. La discrimination et la ségrégation ont trop longtemps infiltré la Vie Américaine. Elles menacent maintenant le futur de chaque Américain. Si elle veut se poursuivre, notre évolution présente devra enrayer la polarisation continue de la communauté américaine et en définitive la destruction des valeurs démocratiques fondamentales ${ }^{3}$.

A l'époque dans son analyse des causes des émeutes raciales la Commission a isolé deux grandes catégories de facteurs. Le premier groupe lie les causes de ces émeutes à la misère économique, alors que l'autre groupe établit un lien avec le changement d'attitude de la communauté noire à l'égard de l'injustice socio-économique. Dans le premier groupe la Commission intègre les trois conditions suivantes, résultats d'une longue situation du préjudice blanc à l'égard des Noirs: Généralisation de la discrimination et de la ségrégation: dans le domaine de l'emploi, l'éducation, le logement, qui résultent de la continuelle exclusion d'un grand nombre de Noirs des bénéfices du progrès économique. La migration noire et l'exode blanc: lesquels ont conduit à de massives et croissantes concentrations de Noirs pauvres dans nos grandes villes, provoquant une crise croissante liée à la détérioration des aménagements, des installations et des services..... Les ghettos noirs: où la ségrégation et la misère qui touchent les jeunes détruisent toute opportunité et poussent à l'échec. Crime, toxicomanie, dépendance à l'égard des services sociaux, amertume ressentiment envers la société en générale et des Blancs en particulier, en sont les résultats ${ }^{4}$. La Commission Kerner faisait remarquer que le sentiment de frustration présent au sein de la communauté noire était la conséquence de ces conditions physiques de vie accentuées par la télévision et les média : Les espoirs déçus sont le résidu des attentes inassouvies réveillées par les grandes victoires juridiques et législatives du mouvement pour les droits civiques et la lutte épique pour l'égalité des droits au Sud. Un climat, qui pousse à l'approbation et à l'encouragement de la violence comme une forme de protestation, a été créé : par le terrorisme dirigé contre la protestation non-violente ; par une défiance ouverte à la loi et aux autorités fédérales des fonctionnaires étatiques et locaux opposant une résistance à la déségrégation; et par quelques groupes protestataires engagés dans la désobéissance civile qui ont, tourné le dos à la non violence, outrepassé les droits constitutionnellement protégés de pétition et de libre réunion, et ont eu recours à la violence pour tenter d'obtenir par la force une modification des lois et des politiques avec lesquelles ils n'étaient pas d'accord. La frustration liée à l'impuissance a conduit nombre de Noirs à être convaincus qu'il n'existait pas d'autre alternative efficace que la violence comme moyen de réparation de leurs griefs et pour faire "bouger le système". Ces frustrations sont reflétées dans la désaffection et l'hostilité à l'égard des institutions de la loi et du gouvernement et de la société blanche qui les contrôle, et l'ampleur de la conscience de race et de solidarité se reflète dans le slogan "Pouvoir Noir". Un nouveau sentiment a surgi parmi les Noirs notamment les jeunes, 
au sein duquel l'amour propre et l'affirmation de l'orgueil racial remplacent l'apathie et la soumission "au système". Les polices ne sont pas simplement le facteur "déclencheur". Pour nombre de Noirs les polices symbolisent le pouvoir blanc, le racisme blanc, la répression blanche. En outre, beaucoup de polices reflètent et expriment les attitudes des Blancs. L'atmosphère d'hostilité et de cynisme est renforcée par la croyance répandue chez les Noirs en l'existence d'une brutalité policière et "de deux niveaux" de justice et de protection, un pour les Noirs et un pour les Blancs. Comme la sagesse populaire et les hypothèses les plus classiques reliaient la violence collective à la privation causée par la "frustration" économique, il n'y eut pas de raison apparente de questionner la validité de cette lugubre prédiction. Il est intéressant pourtant de remarquer qu'un quart de siècle plus tard, la Commission Webster constituée à la suite des émeutes de Los Angeles de 1992 est parvenue aux mêmes conclusions sur l'imminence de la révolte généralisée.

Mais qu'en est-il ? Se dirige-t-on vers une révolte de tous les Noirs Américains? Si nous regardons le monde d'aujourd'hui, Les exemples de violence ethnique demeurent encore trop fréquents. Certains de ces conflits sont d'origine relativement récente (les Sikhs en Inde, les Tibétains en Chine) mais ceux qui s'inscrivent dans une histoire longue et tourmentée sont encore bien plus nombreux (l'IRA en Irlande du nord, les Basques en France et en Espagne, les Arméniens dans l'ex-Union Soviétique, en Iran et en Turquie, les Arabes en Israël, les Tamouls et les Cinghalais au Sri Lanka, etc.). Quoiqu'il en soit, contrastant avec les jours tumultueux des années soixante, les relations inter-raciales aux Etats-Unis, sont apparues avant les émeutes de Los Angeles comme ayant atteint un état de "d'équilibre" maîtrisé. Ce n'est certes pas l'unique exemple d'aboutissement et d'apaisement dans l'histoire. Il existe d'autres cas, comme au Nigéria (avec le conflit entre les Ibos et les Houbas) ou dans certaines parties de l'Inde (le conflit entre les Hindous et les Musulmans, notamment le Bengale Ouest) où après une période de confrontation ouverte extrême, les groupes rivaux ont pu parvenir à un certain degré d'harmonie sociale. Depuis les récentes émeutes on s'interroge. Bien qu'elles aient démontré la profondeur de la frustration au sein de l'Amérique urbaine, elles constituent plus une indication du degré de colère liée à la frustration qu'une manifestation destinée à marquer d'une certaine manière l'anniversaire de l'existence d'un mouvement politique organisé comme l'IRA en Irlande du Nord ou le mouvement nationaliste basque en Espagne. Par conséquent, l'objet de cet article est de rechercher quelques réponses au paradoxe de la situation américaine à savoir pourquoi, contrairement à beaucoup d'autres pays dans le monde, les Etats-Unis ont pu négliger à ce point le problème posé par l'hostilité raciale ouverte même lorsqu'ils étaient confrontés à la détérioration des conditions économiques du membre moyen de la communauté Afro-américaine? Nous démontrerons dans une première partie que la situation économique de la communauté afro-américaine ne s'est pas améliorée et s'est peut-être même détériorée depuis les grandes émeutes des années soixante. Si l'on reprend donc les hypothèses de la frustration-agression dans les théories de la révolte collective, les forces de la révolte auraient du s'intensifier avec cette détérioration. Nous tenterons de répondre dans une deuxième partie à ce paradoxe avec l'aide du modèle modifié du choix rationnel de participation. Contrairement au courant néoclassique des sciences économiques basé sur les modèles $\mathrm{du}$ choix rationnel, ce modèle élargit l'hypothèse fondamentale de la maximisation de l'utilité et prend en compte les facteur d'utilité du groupe. Ce modèle conclut que l'existence de frustration n'est seulement qu'une condition nécessaire pour qu'il y ait 
révolte collective. La condition suffisante nécessite obligatoirement l'existence d'une forte identité politique ou de groupe. Nous discuterons, dans une troisième partie, de la question de l'identité politique des Afro-américains, identité qui s'est affaiblie depuis les journées de violence du milieu des années soixante. En conclusion nous intégrerons ce problème dans une perspective comparative, et nous poserons, pour la forme, la question de savoir si les Etats-Unis ont quelque chose à enseigner au reste du monde, ou s'ils ne doivent être effrayés par leur expérience malheureuse.

\section{L'ETAT ACTUEL DE L'AFRO-AMERIQUE : LA FORME DU PARADOXE}

Si l'estimation de la situation économique des Afro-américains était en baisse dans les années soixante, le changement intervenu durant les années soixante dix et quatrevingt a été tout simplement catastrophique. Ainsi, le National Research Council, dans un rapport publié en 1989, conclut sans équivoque que «Les plus grands profits économiques pour les Noirs ont été obtenus entre 1940 et 1960. Depuis le début des années soixante dix, le statut économique des Noirs par rapport aux Blancs a, en moyenne, stagné ou s'est détérioré » ${ }^{5}$. Les statistiques suggèrent que même si nombre de noirs, en bénéficiant de l'amélioration de leur environnement social et politique, ont grandement profité des périodes de lente, mais soutenue, expansion économique, la condition économique d'une grande partie de la population afro-américaine s'est détériorée et souvent de façon considérable. Le revenu personnel Pour comprendre dans un premier temps la situation économique de la communauté afro-américaine, nous pouvons considérer le rapport existant entre le salaire potentiel des Noirs et celui des Blancs. Selon une étude de Current Population datant de 1985 (figure 1, page 42 ), en 1969 un homme noir moyen gagnait 68,1 cents pour chaque dollar gagné par un blanc moyen. Ce chiffre est passé modestement à 72.5 cents en 1985, soit une augmentation de $6,5 \%$ en 15 ans. Ce salaire, plutôt modeste, était basé sur le fait qu'un individu était rémunéré et donc qu'il travaillait. Cependant si l'on prenait la population entière pour calculer le revenu escompté possible sur une vie entière, les Noirs ne pouvaient espérer gagner, en 1967, que l'équivalent de $56.7 \%$ des revenus gagnés par un blanc à situation égale au cours de sa vie. En 1984, ce chiffre est tombé à $56.1 \%$, enregistrant une perte de $1,1 \%$. Ces chiffres indiquent que si un noir a occupé un emploi entre 1969 et 1985 il se retrouverait, dans une relative mesure, dans des conditions légèrement meilleures, rattrapant ainsi son homologue blanc. Quoiqu'il en soit la position d'un enfant noir né entre ces deux années resterait, théoriquement la même. Un enfant né en 1985, enregistrera une réduction plus légère des inégalités qui le sépare des Blancs que s'il était né en 1969.

La pauvreté Les situations de pauvreté et de non scolarisation des Noirs se sont accentuées encore plus nettement au cours de ces vingt années. Ainsi un noir sans diplôme de fin d'études secondaires pouvait espérer gagner 213 dollars la semaine en 1984 (en dollars constant de 1984) alors qu'il pouvait espérer en gagner 312 en 1969, soit une perte vertigineuse de $21 \%$ de promesses de gains. La situation est encore pire pour les femmes noires sans diplôme d'études secondaires. Elles pouvaient espérer gagner 201 dollars en 1969 et 152 dollars en 1984, une chute de 24\% (figure 2 page 42). La situation ne s'est pas améliorée de façon notable pour ceux qui ont eu un accès un peu plus ouvert au système d'éducation. Pour les hommes noirs ayant le niveau du 
collège mais pas le diplôme (degree), le salaire en dollars constant de 1984 est tombé à 315 dollars la semaine en 1984, alors qu'il était de 394 dollars en 1969, une chute de 20 $\%$. Sur la même période la perte de revenu correspondante pour les femmes à 32 \% (de 308 dollars en 1969 à 242 dollars en 1984) ${ }^{6}$. Toutefois selon une étude ${ }^{7}$, durant la même période les revenus des hommes noirs ayant eu une scolarité correcte ont augmenté dans des proportions équivalentes à celles des Blancs. En 1984, un noir ayant suivi une «bonne » scolarité pouvait espérer gagner à situation égale l'équivalent de $74 \%$ de ce gagnait un blanc. D'autres statistiques effrayantes ont été établies par Current Population Survey en 1985 (Voir tableau page 44.). Ce tableau montre indubitablement une augmentation de la concentration du chômage au sein des Afro-américains.

L'éducation L'écart entre les deux races en matière de réussite scolaire est également persistant, et dans certaines zones le fossé s'est élargi. Bien que de récents articles de journaux suggèrent que les Noirs restent plus longtemps au lycée, ces derniers sont désabusés sur leurs réussites dans le secteur de l'éducation secondaire. Alors que l'inscription dans les collèges pour les diplômés d'études secondaires noirs augmentaient de façon aiguë depuis la fin des années soixante, elle a décliné dans le milieu des années soixante dix. Alors que les taux d'inscription des Blancs a recommencé à augmenter celui des Noirs demeure dans les années quatre-vingt bien en deçà de celui des années soixante-dix. La proportion des diplômes délivrés aux Noirs a aussi diminué.

La santé L'augmentation du taux de pauvreté au sein de la population noire a fait beaucoup de victimes, ce n'est pas une surprise, parmi les plus faibles de la communauté, à savoir les enfants, les malades et les personnes âgées. En 1986, 43 \% des enfants de moins de 18 ans demeuraient dans des familles vivant au dessous du seuil de pauvreté. Nombre des difficultés rencontrées par les enfants proviennent de la structure mono-parentale, prédominante, parmi les foyers noirs composés de mères célibataires. Le National Research Council conclut à ce propos que "au cours de leur enfance $86 \%$ des enfants noirs...passent vraisemblablement un certain temps dans un foyer mono-parental"s. Une évaluation comparative des mesures de santé et d'alimentation témoigne d'une histoire similaire.

Le logement La ségrégation à laquelle est confrontée la communauté afro-américaine dans le domaine de l'habitat a pris une tournure différente durant les vingt dernières années. L'adoption de lois relatives aux droits civiques et l'assouplissement général des opinions ont atténué le problème de la ségrégation relative à l'habitat pour les couches défavorisées. Cependant, bien que les rapports du recensement décennal constate l'abandon de la ségrégation dans les grandes villes métropolitaines, ce changement n'a affecté que les familles des classes moyennes et des classes moyennes supérieures noires. Dans une étude récente, Massey et Denton ${ }^{9}$ apportent la preuve de ce qu'ils appellent "l'hyper ségrégation" basée sur la classe économique. On peut donc semble-til conclure sans se tromper qu'une partie relativement importante de noirs a pu quitter ses quartiers pauvres et isolés et s'intégrer avec succès à l'Amérique moyenne. Toutefois pour une grande majorité de noirs la pauvreté et l'isolement se sont accentués. Les statistiques froides donnent rarement une image complète et parfaite. Mais ce que ces chiffres montrent déjà à eux seuls, est suffisamment effrayant. De récents rapports prétendent que l'espérance de vie d'un homme noir à Harlem est plus courte que celle d'un homme vivant au Bangladesh, l'une des nations les plus pauvres sur la terre. Il y a également plus de jeunes noirs sous la protection du système de la 
justice criminelle qu'inscrits dans les collèges. Ces statistiques honteuses posent cependant un problème paradoxal : Où est la révolution? Qu'en est-il des prédictions apocalyptiques de la Commission Kerner et de beaucoup d'autres sociologues. Certains pourraient dire que les récentes émeutes de Los Angeles et Miami sont les signes avantcoureurs d'un futur inquiétant annonçant une violente guerre des races, suivant les lignes de l'Irlande du Nord ou de la Yougoslavie. Toutefois je voudrais discuter dans cet article le fait que même si dans l'Amérique urbaine actuelle toutes les conditions nécessaires sont réunies pour qu'existe un tel mouvement collectif violent, les conditions suffisantes sont, elles, absentes.

\section{LA REBELLION COLLECTIVE : UNE PERSPECTIVE THEORIQUE}

Si l'on remonte dans l'Histoire, on trouve depuis les temps les plus reculés de l'érudition dont les traces demeurent encore à notre disposition, des philosophes et des penseurs du monde social qui tentent de déterminer les raisons pour lesquelles, à certains moments, les gens commettent des actes de violence contre l'ordre politique et social établi, au nom de la race, de la religion, de la langue, ou de l'allégeance politique. Si l'on examine cette longue et complexe histoire des tentatives visant à expliquer la participation à des actes de violence politique, nous nous trouvons en face de deux grandes familles: La première voit dans le déséquilibre et l'inégalité de la structure sociale les facteurs qui donnent naissance à la violence politique. Le second groupe de théories, quant à lui, postule que la motivation pour la participation à un mouvement s'inscrit dans la logique de la poursuite d'un intérêt propre.

Les approches classiques Cependant les deux lignes de raisonnement se heurtent à un problème conceptuel. Les théories qui composent le premier groupe expliquent pourquoi les gens participent à des actions collectives mais n'offrent aucune explication de l'inactivité des gens, même confrontés à l'extrême "frustration". Par contraste, le second groupe de théorie s'embourbe dans le marécage conceptuel de ce que l'on appelle le ticket gratuit. Mancur Olson dans son très célèbre livre "la logique de l'action collective"10 a soulevé la question de savoir : que si les actions collectives sont entreprises par les participants pour obtenir un bien public - le bénéfice devra être redistribué parmi tous les membres du groupe sans se soucier de la participation, tout en sachant que le coût de l'effort pour obtenir le bien public sera supporter par les participants - être participant ne fait donc plus sens économiquement. Par conséquent, un individu rationnel devrait plutôt se comporter comme un free-rider et attendre que les autres se portent volontaires pour obtenir le bien public. Amartya Sen ${ }^{11}$ a, de façon similaire, posé le "problème de l'assurance". Même si dans une communauté tout le monde peut sembler désirer l'existence d'une quelconque action collective, suivant les préceptes de la maximisation du profit individuel, personne ne prendrait l'initiative de l'action. S'inspirant des problèmes du free-ridership, Tullock ${ }^{12}$ pose le paradoxe de la révolution en affirmant que ceux qui proclament prendre part à un mouvement révolutionnaire pour des considérations purement altruistes, sont également irrationnels (ils participent juste pour le plaisir de participer), ou ont un motif caché de profit personnel. Par conséquent, les théories sur la participation basées sur la théorie économique peuvent à elles seules certes, nous donner les raisons pour lesquelles les gens ne prendront pas part à l'action collective et devenant donc des free-riders, mais 
elles sont singulièrement incapables d'expliquer pourquoi en réalité les gens choisissent actuellement de participer ou de s'engager rationnellement dans des actions altruistes. Pour surmonter les difficultés conceptuelles liées aux deux paradigmes théoriques, nous offrons une explication en modifiant les arguments relatifs au choix rationnel sur un point qui concilie les théories sur les hypothèses structurelles de l'action collective ${ }^{13}$. Pour expliquer la participation à l'action collective, nous présupposons que contrairement à l'hypothèse du modèle économique un individu n'est pas exclusivement porter à la maximisation de son utilité propre ${ }^{14}$. En effet, conformément à la caractérisation des socio-psychologues, comme Erik Erikson ${ }^{15}$, Tajfel ${ }^{16}$ et ses collaborateurs, l'identité individuelle peut avoir deux composantes ${ }^{17}$ : celle de l'individu unique, distinctes de toutes les autres, et celle de l'individu pris comme le membre d'un groupe. Nous affirmons que c'est la combinaison de la poursuite de l'utilité individuelle et de celle l'utilité collective qui pousse un individu à participer à des actions collectives et notamment donc à un acte de violence politique.

Une hypothèse de travail : les deux composantes de l'identité Afin d'analyser, dans l'ordre, les motivations pour la participation à un acte de défiance politique ouverte, commençons par le modèle du comportement humain. Dans le modèle économique néoclassique il a été présumé qu'un individu cherche perpétuellement à maximiser son intérêt à l'exclusion de tout autre. Toutefois, et en opposition avec cette vue singulière de la dimension de la nature humaine, nous posons pour principe que la perception que nous avons de nous-mêmes se compose de deux identités différentes. La première source d'identité repose sur le fait que nous sommes des entités uniques, distincts, dans ce monde, de tous les autres. La seconde source de notre identité est liée à la perception que nous avons de nous-même en tant que partie d'un groupe plus grand. L'adhésion à ce groupe peut être attributive, basée sur la naissance, c'est le cas des groupes tels que les groupes ethniques, linguistiques, culturo-religieux, ou d'adoption. L'adhésion à un groupe adoptif n'est pas automatiquement acquise, ou plus précisément, l'individu choisit d'appartenir au groupe. L'adhésion à un groupe tel que Green Peace ou à un groupe anti-avortement ne repose pas sur la naissance mais dépend du penchant de l'individu à s'associer avec des personnes aux positions idéologiques similaires. Nous appartenons tous à des groupes, et, dans presque chaque recherche sur une branche sociale spécifique, les chercheurs ont trouvé des groupes qui savent extrêmement bien motiver les gens pour l'action humaine. En fait, l'existence d'une identité de groupe est la seule solution au problème de l'action collective des free-riders. Sans une forte identification à un groupe, les individus ne trouvent pas de raison rationnelle de participer à des actions collectives. Aucune bataille ne sera jamais gagnée, aucun bien public ne sera jamais obtenu. Par conséquent il n'est guère surprenant, que pour être prête à la bataille, la nouvelle recrue doive subir un entraînement rigoureux de son esprit afin d'immerger son individualisme dans l'identité collective. La force de l'identité d'un groupe passe par l'inculcation de l'idéologie du groupe. Au sein d'une société, le processus s'inscrit dans l'histoire, les folklores et les autres mythes politiques et sociaux que nous aimons tant fabriquer.

Vers un nouveau modèle d'utilité Nous supposerons donc qu'au cours d'une journée, les individus, prennent part à la production de biens et de services, dont ils bénéficient personnellement, comme ils profitent à l'ensemble du groupe. Nous pouvons qualifier le premier type d'activité d'économique ou d'intéressée (sans aucune connotation péjorative) et le second type d'activité politique ou d'intérêt de groupe. Les activités économiques produisent des biens et des services pour la consommation individuelle. 
Nous pouvons mesurer généralement le revenu économique aussi bien en terme absolu qu'en terme relatif. En terme absolu le revenu économique personnel comprend la santé, le bien-être, l'épanouissement, la compétence, le pouvoir, l'affection, la rectitude et la déférence ${ }^{18}$. Le revenu individuel peut aussi être mesuré en terme relatif, évalué par rapport à l'acteur référent du groupe. D'autre part, les revenus générés par les activités politiques, comprennent les biens et les services destinés à la consommation collective (ou biens publics), tels la fin de la discrimination, une révolution couronnée de succès qui transforme entièrement la structure économique et sociale, le renversement d'un gouvernement sans légitimité politique (perçu comme un bien désirable) etc. Ce sont des biens de consommation collective ${ }^{19}$. Expliquons plus précisément les autres composantes de la décision individuelle calculée, basées sur notre modèle: L'obtention d'un bien politique peut apporter la prospérité aux individus qui ont été associés à l'effort qui a permis cette acquisition. Le bénéfice individuel peut prendre la forme de butin provenant d'émeutes ou d'autres actes d'anomie, ou même d'une position personnelle etc., au sein du nouveau régime. L'influence des pairs constitue pour un individu un autre facteur important de motivation à la participation à un mouvement politique. Plusieurs études ont démontré l'importance de cette influence dans le processus de recrutement pour un mouvement révolutionnaire ${ }^{20}$. Il est inutile de préciser que la participation à des activités contre le système n'apporte pas que des bénéfices. Elle est déterminée dans une large mesure par le facteur coût. Le coût d'une participation est fixé par le gouvernement en terme de réactions coercitives de sa part. Nous supposons donc qu'un individu maximise ses intérêts, produit des fruits des biens publics et privés net des coûts de participation aux activités politiques ${ }^{21}$. Par conséquent la décision d'un individu de se joindre à une rébellion pourra se baser sur la perception de cinq traits majeurs :

- a) L'utilité pour le groupe de l'obtention du bien collectif désiré.

- b) L'utilité en termes de gains personnels pécuniaires de la participation à une action collective.

- c) L'opportunité du coût des activités politiques, ou l'inutilité de la perte de ce que l'on aurait pu obtenir par soi-même au sein du système. -d) L'inutilité d'être puni par les autorités pour avoir pris part à des activités dirigées contre le système.

- e) l'inutilité de la pression des pairs ou des menaces de châtiment liées à l'inactivité. Nous soutenons de plus que la perception pour un individu liée à l'obtention d'un bien public pour le groupe dépendra de la préférence pour le bien collectif ou l'idéologie, qui est en rapport direct avec la force de l'identité du groupe. La question est de savoir, maintenant, ce qui déterminera notre choix entre les biens privés ou publics dans la répartition du temps de travail ? Les cinq facteurs cités précédemment peuvent être classés généralement en trois catégories : i) la possibilité d'acquérir des biens privés, ii) la force idéologique qui émane de la perception de l'utilité des biens publics, iii) la possibilité d'une arrestation par les autorités, et la sévérité des peines. Si la possibilité d'obtention d'un bien privé est très élevée (ou plus grande par rapport à l'utilité des biens publics net du coût de participation) un individu sera plus disposé à prendre part à des activités purement économiques, destinées à enrichir sa propre personne. Par conséquent, en général, les riches sont moins disposés à se joindre à des forces révolutionnaires. De même, un taux de croissance économique soutenu, situation qui profite à la plupart et qui déclenche des attentes très fortes pour le futur est plus à même de séparer, d'éloigner la nation d'une ferveur révolutionnaire ${ }^{22}$. Finalement, la décision de se joindre à un mouvement collectif a plus de chance d'être influencée par 
le facteur coût imposé par les autorités. Plus le facteur coût (la probabilité d'arrestation et la sévérité de la punition) est fort, plus l'inclination à rejoindre les forces rebelles est faible. En somme, je postule que la décision d'un individu de se joindre à un mouvement collectif sera déterminée par le coût de son opportunité (et par conséquent de frustration relative), la force de l'identité collective et le coût physique de l'engagement dans un mouvement contre le système. L'individu et l'environnement Nous posons pour principe que la décision d'une personne de se joindre à une rébellion collective est déterminée par sa propre évaluation de l'utilité qu'elle en attend. Evaluation qui, à son tour, est influencée par des facteurs extérieurs, définis comme exogènes au modèle d'utilité attendu. Nous classons ces facteurs dans trois grandes catégories : ceux générés par l'opposition, ceux émanant des autorités et ceux résultant d'événements imprévisibles incontrôlables qui forgent le cours de notre histoire. Ainsi le processus peut-être décrit à l'aide de la figure 8 (page 44): A gauche, nous avons situé les actions délibérées des forces d'opposition; à droite nous avons montré l'influence des autorités. Par ses actions l'opposition tente d'influencer les participants potentiels. Elle essaye d'y parvenir en ayant recours à ce que l'on pourrait grossièrement nommer des facteurs de direction et de coercition. Le facteur de direction peut à son tour être divisé entre la capacité de répandre une idéologie et celle d'administrer et de mobiliser les forces d'opposition. L'autre aspect de leur arsenal résultent de leurs capacités coercitives, par lequel elles peuvent contraindre une personne, obstacle potentiel immobile, à devenir un participant actif. Les forces environnementales du coté du gouvernement résident aussi dans la perception de sa légitimité et dans sa capacité à imposer des sanctions négatives aux dissidents. Comme le montre la figure, l'impact des facteurs environnementaux sous contrôle de l'opposition s'ajoutent de fait aux causes de l'insurrection alors que ceux influencés par l'action gouvernementale sont destinés à réduire le niveau du conflit et à maintenir le statu quo. Au bas de la figure, nous avons placé les événements précédents. C'est une généralisation des significations données aux "événements précipitants" de Smelser ${ }^{23}$. Nous n'aurons pas ici recours à cette expression car les résultats de ces événements imprévisibles peuvent conduire dans des directions opposées, soit en améliorant, soit en exacerbant une situation conflictuelle. Ainsi, un événement aussi particulier que le refus de Rosa Park de s'asseoir au fond d'un bus, peut devenir un facteur de cristallisation, l'appel au combat d'un mouvement. De même, un événement au cours duquel un gouvernement effectue certaines démarches de conciliation en période de tension, peut réduire le niveau de conflit dans une société. La direction tend à affecter le comportement et la participation des gens de deux façons. Elle exacerbe l'intérêt des biens publics attendus. Les facteurs de direction influencent la perception que la population a de l'utilité d'un bien public, ce que l'on nomme le facteur idéologique. L'autre aspect de la direction réside dans la capacité à mobiliser les forces d'opposition. Avec de tout côté un niveau de participation plus important il contribue à créer une atmosphère dans laquelle les personnes qui font obstacles sont capables de sousestimer la probabilité d'être appréhendées et sont donc tentées de participer à des actes de rébellion. De même, un individu peut, avec un plus grand niveau de mobilisation, percevoir le fait que ces exigences politiques ont une plus grande chance d'être satisfaites. En conséquence, selon son évaluation, la productivité marginale de son époque à générer des biens politiques augmentera. En outre le facteur d'équilibre dans la répartition de la participation, est joué par la capacité des autorités à écraser une rébellion en appréhendant et punissant les auteurs. Nous disons donc que les 
motifs tant de participation que de non participation sont nombreux. De fait, le paradoxe de la révolution aux Etats-Unis peut être compris à la lumière de notre modèle par l'analyse du comportement des participants potentiels. Un modèle descriptif peut-être utilisé dans la formulation de certaines hypothèses: La carence économique seule ne mènera pas à un mouvement politique. Il faut donc qu'une forte identité collective existe, pour qu'un mouvement politique prenne racine. La participation déclinera si la perspective d'un revenu individuel devient plus sûre que celle des biens publics. Nous devons nous attendre par conséquent à une participation plus faible de la part des couches sociales élevées. Nous pouvons, au contraire, nous attendre à une plus grande participation des plus jeunes, s'ils ont peu d'avantages économiques à remettre en question. Une évolution de la perception de la capacité à aménager le temps pour produire un bien en plus grande quantité impliquera un effort de production plus grand. Il en résulte une participation politique plus faible en période de prospérité économique. Cette situation a pour corollaire une réévaluation, dans les périodes plus tourmentées, de l'action politique par une population plus importante. On observera par ailleurs ici, un effet boule de neige sur la scène politique. Le rôle des instances dirigeantes de l'opposition sera d'exacerber l'orientation politique des participants potentiels. En conséquence, l'émergence d'une direction politique effective augmentera le niveau de participation politique. Une augmentation du coût de participation tendra à réduire le niveau de participation politique. Un plus grand recours aux forces de sécurité et aux poursuites plus systématiques atténuera donc la violence politique ${ }^{24}$.

\section{RESOLUTION DE L'ENIGME : LE CAS DE L'AFFAIBLISSEMENT D'UNE IDENTITE COLLECTIVE}

Le changement qui s'est opéré dans les relations raciales aux Etats-Unis durant le dernier quart de siècle est évident même pour un observateur non averti. Elles sont bien plus complexes qu'elles ne l'étaient il y a 25 ans. Nous rencontrons toujours, d'une part, les visages pleins de colère des jeunes livrés à eux-mêmes et au crime dans les ghettos urbains, qui seraient prêts à prendre part à la destruction de leur propre voisinage sans aucune retenue. Toutefois, les athlètes noirs des jeux olympiques brandissant leur poing serré au moment de la victoire ont disparu depuis longtemps. Ils ont été remplacés par ceux qui courent derrière le drapeau américain autour du stade olympique, dans un même élan de patriotisme. Même les dirigeants noirs les plus radicaux qui prônent la guerre contre un "système dominé par les blancs" paraissent plus exaltés que menaçants. Afin de mieux comprendre cette transformation je prétends qu'il est essentiel, non seulement, de comprendre pourquoi les gens se rebellent mais aussi d'analyser les raisons qui font qu'ils choisissent de rester en retrait de toute action. Par conséquent, nous nous appliquerons dans la partie suivante à expliquer les motivations qui poussent des Afro-américains à ne pas prendre part à un mouvement politique contre le système. Nous nous appuierons pour cela sur les divers facteurs de décision individuelle à partir de notre modèle. Dans notre modèle comportemental nous avons souligné la nécessité d'une identité collective comme base de rébellion. Nous avançons donc deux raisons majeures au fait que la communauté Afro-américaine de ce pays n'a pas explosé en une rébellion ouverte contre le système politique et économique actuel. Premièrement, elle n'a plus d'identité collective (ou politique) ce qui requiert une définition claire du "nous" contre le "eux" et des termes 
"d'alliés" et "d'ennemis"25. Deuxièmement, les efforts gouvernementaux visant à supprimer un radicalisme politique, par des initiatives législatives comme l'Omnibus Crime Control Act de 1968 et des amendements ultérieurs, a abouti à une telle augmentation des coûts d'organisation qu'il n'a plus été possible pour les radicaux de se constituer en organisations effectives. Par conséquent, en dépit d'une frustration réelle au sein de la communauté noire, celle-ci n'est pas systématiquement canalisée vers l'action politique légale démocratique et non-violente ou vers un radicalisme politique. Le retour sur l'histoire Le lecteur de l'histoire des Afro-américains est souvent frappé par la brutalité du système et par la totale soumission physique, économique, politique et même psychologique d'une race à une autre. La domination complète des Blancs sur les Noirs peut seulement être comparée au système de castes hindou dans lequel les "intouchables" furent assujettis à la caste dominante pendant au moins deux mille ans. A cause de cette répression chacun des deux groupes n'a pu, jusqu'à il y a encore peu de temps, développer une réelle cohésion de l'identité collective pour faire face au système oppressif. Dès les premiers temps de la colonisation, un effort systématique de destruction de l'identité des esclaves a été entrepris. Ils furent non seulement dispersés dans toutes les colonies, de manière à détruire les groupes tribaux et linguistiques mais aussi empêchés d'avoir des liens familiaux normaux afin de limiter leur population ${ }^{26}$. A l'inverse des pays d'Amérique du Sud, où les plantations étaient étendues, la proportion des esclaves par rapport aux non esclaves très forte, les plantations du sud des Etats-Unis ne contribuaient pas à une rébellion ouverte et massive des esclaves. Ajoutons que le Sud de l'après guerre civile, à cause d'un manque de pouvoir économique et de ressources de la communauté afroaméricaine, associé au manque d'intérêt du Nord pour le soutien d'une campagne d'émancipation économique et politique, a vu la résurgence d'une division raciale basée sur les lois de Jim Crow. La communauté afro-américaine démunie de tout pouvoir a été affectée dans ses capacités à constituer une pression politique propre. Les lendemains de la guerre civile furent caractérisés par une érosion rapide de l'identité politique des Noirs, qui n'était à ce moment qu'à l'état embryonnaire. L'identité politique des Noirs entra dans une seconde phase à partir du dernier quart du XIXème siècle et ce jusqu'à la fin du New Deal ${ }^{27}$. Durant cette période le conflit entre Noirs et blancs fut axé sur la vengeance des classes ouvrières blanches à l'encontre de l'empiétement économique des Noirs. Les Noirs étaient souvent utilisés pour remplacer les Blancs qui faisaient grève. Combiné à une migration à grande échelle loin du Sud rural et répressif, cet élément intensifia la tension raciale dans de nombreuses zones urbaines du Nord. Dans l'immédiate après guerre, les relations entre Noirs et blancs ont été marquées par le développement croissant du sens l'identité noire, qui s'est cristallisée pendant les années $60-70^{28}$. Le caractère du conflit a évolué. Le mouvement noir d'après guerre est devenu de plus en plus politique. Durant les deux conflits mondiaux et plus particulièrement pendant la seconde guerre mondiale, la position économique des Noirs a connu un changement réel et ce probablement pour la première fois dans l'histoire américaine. Comme de plus en plus d'hommes ont été appelés en service actif et qu'au même moment le secteur industriel a enregistré une rapide expansion, une demande importante de main-d'oeuvre spécialisée ou non s'est manifesté. Un nombre relativement important de noirs ont profité de ce changement des conditions socioéconomiques. De même, alors que les noirs commençaient à intégrer l'armée américaine, leur perception de la société en général et des relations raciales en particulier commencèrent à changer. Par conséquent, à la suite de l'effort de guerre et 
du changement de nature de l'économie, les Noirs ont commencé à abandonner leur secteur traditionnel d'activité : l'agriculture, travaillant en tant qu'ouvriers agricoles non spécialisés. Le déplacement démographique des zones agricoles et rurales vers l'industrie urbaine donna naissance à de nouveaux ethos culturels et politiques au sein de la nation. Concernant la communauté afro-américaine, l'impact le plus important de ce bouleversement total fut la naissance d'une nouvelle classe moyenne.

Identité collective et structure de classes La classe moyenne a toujours été le moteur de changements sociaux au cours de l'histoire. Le mouvement en faveur des droits civiques dans le sud fut essentiellement un mouvement issu de la classe moyenne noire qui, aux vues de la discrimination ouvertement mesquine, se voyait privée d'une place qui lui revenait à juste titre dans la société. Ces barrières raciales étaient beaucoup plus coûteuses pour cette dernière que pour les noirs pauvres qui vivaient au marge de la société urbaine ou en zones rurales. Si l'on suit notre raisonnement économique, nous pouvons constater que le mouvement noir a donc pris racine dans les classes moyennes, et plus particulièrement chez les pasteurs noirs beaucoup plus impliqués idéologiquement, et dont la subsistance ne dépend pas de la société extérieure. C'est eux qui menèrent le combat pour l'égalité raciale et la fin de la discrimination. Ils furent aidés dans leurs démarches par les plus brillants jeunes hommes et femmes de la communauté, avec un très faible coût, habités par un sens aigu des aspirations bloquées et confrontées à l'augmentation de leurs niveaux d'espérances avec un très haut degré d'engagement idéologique ${ }^{29}$. Les émeutes observées dans le Nord furent légèrement différentes. La non-existence de pratiques discriminatoires manifestes a influé sur les expressions de frustration qui prirent une autre forme que dans le sud. Au nord, la frustration et la colère revêtirent moins cet aspect politique et se manifestèrent plutôt sous la forme d'émeutes. Si nous observons maintenant les motivations premières des gens qui participent à des manifestations pacifiques, on remarque qu'ils n'agissent pas véritablement dans une optique d'obtention de gains financiers. Leur motivation première se doit donc d'être idéologique, plus spécialement quand la perspective de succès du mouvement est plus que douteuse. D'autre part les émeutes ont attiré une grande variétés de participants. Au cours d'une situation d'émeutes on trouve indubitablement les idéologues qui tendent à attaquer les soi-disant «symboles d'oppression ». On trouve également des personnes mues par d'autres motifs, tels que des mercenaires ou des éléments criminels qui tirent profit du désordre.

Identité collective et situation émeutière Dans une situation d'émeutes, on peut aussi rencontrer ceux qui selon Tullock ${ }^{30}$ et Banfield ${ }^{31}$, ont pour but de se distraire. Ainsi de par leur nature même, les émeutes urbaines vont de pair avec la motivation politique $\mathrm{du}$ «Nouvel homme du Ghetto» ou du «Noir Urbain» et des chercheurs "d'amusements et de profit " ${ }^{32}$ : quoiqu'il en soit, ayant atteint son apogée cette forte prise de conscience d'une identité raciale a commencé à vaciller à partir de la fin des années 1970. A titre d'exemple, la plupart des gens (qui par ailleurs étaient de races différentes) ayant pris part aux émeutes de Los Angeles n'avaient aucune aspiration politique. Tout comme la foule de Gustave Le Bon ils ont simplement laissé libre cours à l'épanchement de frustrations ou tiré profit de l'absence d'une force policière effective et dissuasive ${ }^{33}$. Nous prétendons que cette érosion de l'identité collective a été l'un des motifs majeurs du manque de cohésion dans le mouvement de violence collective et ethnique aux Etats-Unis. Essayons maintenant d'analyser certaines des raisons de l'évolution de l'identité noire ces dernières années. 
Les facteurs du « Nous " La perception d'une identité noire aura été d'assez courte durée. Commençant à la fin des années soixante, la communauté perdit un atout primordial de motivation: les définitions $d u$ « Nous » et de «l'autre ». L'absence d'un « nous » clairement défini est liée à la fragmentation extrême de la communauté noire. Cette fragmentation économique s'est manifestée dans le schéma évolutif de l'emploi et donc dans la distribution au sein de chaque groupe racial. A partir des années soixante, les hommes et les femmes noires ont connu une augmentation soutenue de leurs positions parmi les cols blancs et dans les activités professionnelles (voir la figure 3, page 42). Quoi qu'il en soit cette croissance observée chez les cols blancs a s'est limtée à une faible minorité de noirs. Le reste de la population continue à se morfondre dans des métiers mal rémunérés ou se retrouve tout simplement au chômage. Le résultat de cette disparité : un abîme au sein même de la communauté, bien plus profond que ce qui existe dans le reste du pays (voir fig. 4 et 5, pages 42 et 43 ). La figure 4 présente le pourcentage de noirs qui ont gagné moins de 10000 dollars, entre 25 et 35000 dollars et plus de 75000 dollars au cours du dollar de 1988 puis recalculé par rapport à 1967 . Comme nous pouvons le constater, alors que les chiffres concernant les Noirs gagnant moins de 10000 dollars ont accusé une légère baisse, le pourcentage concernant les revenus moyens les plus bas sont restés en pratique identiques. Le pourcentage de noirs gagnant plus de 75000 dollars a changé. La figure reflète ce changement total parmi les différents groupes de revenus entre 1967 et 1988 . Remarquons ici que la position relative des revenus des noirs en général est demeurée à peu près inchangée durant les vingt dernières, ceux qui ont accédés aux couches aisées de la classe moyenne ou de la bourgeoisie ayant particulièrement réussi. Malgré des efforts massifs entrepris grâce aux législations sur les droits civiques et les programmes de la « Grande société », il demeure particulièrement troublant que seul $20 \%$ approximativement de la population noire ait pu jusque là profiter de ces actions de façon tangible ${ }^{34}$. Nous considérons que cette fragmentation extrême de la structure des classes au sein de la communauté afro-américaine a laissé cette dernière sans aucun sentiment d'identité partagée. Ajoutons-y l'élément "d'hyper-ségrégation " ${ }^{35}$, le niveau d'isolement pour une grande majorité de noirs par rapport au reste de la société aussi bien que par rapport aux plus riches de leurs congénères. Cette fragmentation a posé quelques problèmes singuliers à la participation. Pour ceux qui trouvèrent leur place au sein du "rêve américain ", prendre part à un mouvement n'avait pas de sens. Alors que pour ceux abandonnés dans les ghettos, la solution la plus simple résidait dans le trafic lucratif de la drogue. Le mouvement politique noir voyait ainsi son allié le plus naturel, la jeunesse, pivot de tout mouvement politique, dénigré : Cette jeunesse ne témoignait que faiblement d'une idéologie. La nature de la société évoluant, les changements nécessaires devenaient trop complexes. Par conséquent, pour ces jeunes, à l'inverse de leurs prédécesseurs, le fait de rejoindre un mouvement politique n'avait pas de sens (économique ou autre).

Les facteurs du «Eux " Le protagoniste du dessin animé Pogo avait un problème célèbre, il ne pouvait identifier son ennemi. Ainsi donc pour ressentir un "nous" identifiable, nous avons besoin de nous référer à un " eux » reconnaissable. L'un des problèmes majeurs de la communauté noire fut la perte d'un ennemi identifiable ${ }^{36}$. Dans un sondage récent effectué auprès d'afro-américains après les émeutes, $51 \%$ des personnes interrogées étaient d'accord avec l'idée que sur les dix dernières années, la qualité de vie des Noirs avait empiré, $46 \%$ pensaient que la solution à ce problème résidait dans les individus eux-mêmes et seuls 7 à $16 \%$ associaient le racisme des Blancs 
aux divers problèmes sociaux auxquels leur communauté devait faire face ${ }^{37}$. Pourquoi un tel changement? Pour la communauté noire, les oppresseurs se caractérisaient dans le passé par des signes de discrimination évidente, les forces de police constituées presque uniquement de blancs et le symbole du pouvoir: les tribunaux et mairies exclusivement administrés par les blancs. La Commission Kerner a justement souligné que : «La Crime Commission Police Task Force a pensé que le fait d'avoir une force de police à forte proportion blanche dans un quartier noir pouvait opérer comme un dangereux facteur d'irritation; un sentiment pouvait se développer à savoir que la communauté n'a pas assuré la fonction de police pour maintenir le statu quo. Il est prouvé que les officiers noirs ${ }^{38}$ peuvent être particulièrement efficace dans le contrôle des troubles qui éclatent. Après avoir étudié les bons résultats obtenus par l'armée et la garde nationale au cours des troubles de Détroit, nous avons conclu que le plus grand pourcentage de noirs dans l'armée avait contribué de façon substantielle à l'obtention de ces meilleurs résultats" ${ }^{19}$. La Commission a contacté bon nombre de départements de police et seuls 28 ont répondu aux questions relatives à la composition de leurs forces. L'éventail des officiers de police assermentés, au sein de ce groupe, est susceptible d'influencer dans le sens d'une exagération la moyenne nationale, il allait de 1 à $21 \%$ des forces de police. Il devenait sans fin dès qu'on le comparait à la démographie mixte de ces villes. Sur les 28 villes, 16 (plus de $57 \%$ ) avaient une proportion de d'officiers noirs inférieure à $20 \%$ par rapport à la population noire. Seuls $2 \%$ des départements présentaient un nombre d'officiers noirs excédant la moitié de la proportion démographique de la population noire dans la ville. Inutile de dire que la proportion d'officiers était quasi minuscule. Depuis le rapport de la Commission Kerner, la peur des désordres sociaux et l'adoption de l'Equal Employment Opportunity Act, les noirs ont une bien meilleure image des forces de police de la nation. Dans un rapport récent, William Lewis ${ }^{40}(1987)$ a étudié 46 villes en 1975 et 72 en 1985. Il a comparé le pourcentage de noirs au sein de chaque département de police à la proportion conforme à l'idée d'égalité. Cette comparaison a fait à son tour l'objet d'une classification en quatre catégories: un indice de 0.75 ou plus est considéré comme "hautement conforme"; un indice situé en 0.50 et 0.75 est perçu comme "moyennement conforme" ; un indice situé entre 0.25 et 0.50 est considéré comme "peu conforme" ; un de 0.25 ou moins est considéré comme "non conforme" ${ }^{41}$. Nous avons reconstitué le pourcentage des villes qui tombaient dans la catégorie "moyennement conforme" et mieux encore combiné ces données à celles fournies par la Commission Kerner. L'amélioration presque caricaturale du recrutement des noirs depuis les années soixante est décrite dans la figure 6 (page 43) ${ }^{42}$. A l'inverse, la représentation noire dans le corps judiciaire n'a pas suivi le rythme de leur proportion démographique. Quoiqu'il en soit, les gains des Noirs ont également été impressionnants si l'on considère qu'au début des années soixante, leur représentation au sein des tribunaux américains se limitait aux emplois de portiers et de concierges $^{43}$. De même si l'on observe les plus grandes métropoles, nous remarquons que beaucoup d'entre elles ont élu à des postes de maires ou à d'autres postes officiels électifs des noirs. La représentation au niveau des plus hautes sphères l'Etat se met en oeuvre rapidement. Nous pensons donc que l'afflux de personnels judiciaires et de fonctionnaires noirs chargés de l'application des lois privent les Noirs de leur ennemi facilement identifiable.

Coercition gouvernementale : un facteur coût croissant Il est difficile de rendre compte par écrit de la perte de la prédominance idéologique. Néanmoins il parait 
presque évident que l'assassinat de Martin Luther King asséna un coup fatal au mouvement noir. Personne au sein des mouvements noirs ne pouvait assurer une relève digne du docteur King. Face à l'opposition politique croissante, la nation adopta l'Omnibus Crime Control and Safe Street Act en 1968, qui fut amendée ultérieurement durant l'administration Nixon. A la suite de cette série de lois, les efforts dans le domaine de l'application de la loi fédérale connurent un nouvel essor. Des budgets furent attribués à la sécurité intérieure (figure 7, page 43) et accompagnés par des plans élaborés pour la suppression du radicalisme politique. Les nouvelles technologies permettaient une meilleure surveillance et des arrestations dans de meilleures conditions. Les connaissances acquises en matière d'affrontements politiques, fournirent de nouvelles armes aux forces de police pour mieux écraser les mouvements dissidents possédant quelque potentiel pour défier le système politique établi. Le sociologue Gary Marx ${ }^{44}$ a contribué en grande partie à la compréhension des diverses stratégies de contre-insurrection adoptées par la police pour mieux déstabiliser les mouvements politiques. Ainsi souligne-t-i ${ }^{45}$, que par l'infiltration du mouvement la police peut décider d'une douzaine d'actions stratégiques : a) inhiber la capacité d'action corporatiste b) diriger les énergies du mouvement vers l'adoption de position de défense pour le maintien des biens acquis afin de ne pas le voir poursuivre des buts relatif à des changements sociaux plus étendus. c) créer une image publique défavorable pour mieux contrer l'idéologie. d) Rassembler les informations concernant le mouvement pour arrêter et poursuivre les participants. e) Empêcher l'approvisionnement financier et matériel. f) empêcher la liberté de mouvement, d'expression et d'action; créer un mythe de la surveillance et de la répression; appliquer les sanctions légales. g) Saper le moral des participants. h) engager un processus de débauchage i) éliminer et déplacer les meneurs j) encourager les conflits internes $\mathrm{k}$ ) encourager les conflits externes avec les alliés potentiels et les opposants. l) entraver et saboter des actions spécifiques. Il est bien entendu presque impossible de préciser l'étendue complète d'une opération d'infiltration d'un mouvement, toutefois l'augmentation précipitée des dépenses de sécurité intérieure après le milieu des années soixante constitue un indice notable de l'importance accordée à la surveillance et à l'interdiction par les différentes administrations. Il parait évident que face à de tels efforts, les organisations radicales noires n'étaient guère de taille. Elles subirent une défaite militaire qui leur fut fatale et dont elles ne se sont toujours pas remises.

\section{CONCLUSION}

\section{Les relations inter-raciales dans une perspective mondiale}

La tranquillité relative des relations inter-raciales aux Etats-Unis établit un contraste éclatant avec de nombreux pays dans le monde. Même le Canada, réputé pour son climat social paisible fait face à la sinistre perspective d'une explosion. L'ex-Union Soviétique, en dépit de sa prétendue politique d'égalité, conduite pendant 70 ans, est en train de réaliser à quel point les troubles ethniques, culturels, et religieux peuvent être difficiles à gérer. Les pays les moins avancés, tels que le Sri Lanka, après des décennies de tranquillité politique et d'harmonie raciale sont à leur tour pris dans le cercle vicieux de la violence ethnique. La Grande-Bretagne n'est pas prête de résoudre le problème irlandais. Ainsi nous pouvons légitimement nous poser la question de savoir 
si les Etats-Unis ont quelque enseignement important à délivrer au reste du monde où si ces derniers doit être effrayée par leur expérience malheureuse ? En réponse à cette question, regardons les quelques éléments communs à toute violence ethnique. Nous avons souligné le fait que pour aboutir à un mouvement collectif il faut avant tout une identité collective. Ce sentiment repose sur deux aspects: la cohésion intracommunautaire et le degré de différences entre les groupes. Dans le premier cas, l'identité collective dépend du degré de proximité que ressentent les individus membres d'un même groupe ethnique. Dans le second cas, elle dépendra de l'ampleur de la distinction qu'effectue le sous-groupe par rapport au groupe national. Etant donné que la plupart des hypothèses ne sont pas mesurables en terme quantitatifs, ce qui est tout fait compréhensible, la recherche empirique ne s'est guère développée dans la perspective d'une distinction des facteurs qui contribuent à l'approfondissement des deux notions. Néanmoins, nous pouvons supposer que la cohésion du groupe dépendra du "Nous" commun. Elle sera susceptible d'acquérir une force si le groupe considéré est confiné en grande partie dans des limites territoriales définies, si sa structure socioéconomique est uniforme, s'il possède un langage commun, un héritage culturel, religieux ou ethnique et enfin, dernier élément mais non le moindre, s'il a une origine culturelle qui promeut la cohésion du groupe. L'intensité de la passion de la différence interne au groupe dépendra de la facilité avec laquelle un groupe peut identifier un ennemi commun responsable de ce sentiment de "frustration". Si nous comparons maintenant les Afro-américains avec les catholiques d'Irlande du nord ou les Noirs d'Afrique du sud, une différence apparaît : le manque de cohésion de la communauté noire aux Etats-Unis. En Irlande du nord, les catholiques sont obligés de rester dans des limites géographiques relativement bien définies, d'avoir des niveaux de vie socioéconomique similaires et de pratiquer une même religion. Les Noirs aux Etats-Unis, n'ont au contraire pas de limite géographique bien précise et ils ne peuvent revendiquer un territoire comme leur "terre natale". Ils n'ont pas la même religion. L'existence d'un langage les différenciant du groupe dominant est, au mieux, un problème complexe qui ne parait pas fournir une base solide à la différenciation des groupes qui aboutit à l'isolement social. La formation d'une identité de groupe cohésive dans les vieux quartiers d'une ville devient encore plus compliquée dès lors que de nouveaux immigrants intègrent le groupe. Liés par la misère commune, il est possible de maintenir des hostilités locales et une violence des gangs parmi les groupes ethnolinguistique, mais cet ingrédient ne permet pas d'insurrection à grande échelle ou la formation d'un mouvement politique. Les facteurs culturels de cohésion constituent par ailleurs un champ d'études encore peu exploré. L'éloge de l'individualisme a toujours caractérisé l'ethos américain et ce depuis les premiers temps de la colonisation. Ceci explique que l'aile gauche de la politique radicale n'a jamais été très populaire aux Etats-Unis même durant l'abîme de la grande dépression. Ainsi les enfants noirs, à l'inverse des enfants originaires d'autres parties du monde ont grandi avec l'idée de la notion de réussite personnelle. Aussi ils auraient donc, selon notre modèle comportemental, tendance à avoir une préférence plus faible pour les biens collectifs. Quand aux différences entre groupes, nous pouvons voir que l'identification de l'ennemi est bien plus facile dans les nombreux autres pays confrontés à une violence ethnique. Ainsi en Irlande du nord les forces de police sont composées dans leur quasi-totalité de protestants. Le même schéma se reproduit au sein de l'armée britannique considérée comme rien de moins qu'une force d'occupation. Les Sikhs en Inde, Les Tamouls et Cinghalais au Sri Lanka, les Noirs en Afrique du Sud, les Basques en 
Espagne, les néo-nazis en Allemagne, pour donner seulement quelques exemples, peuvent facilement identifier leur groupe ennemi au contraire des Noirs américains. Depuis que les Noirs ont été intégrés au sein du système judiciaire, à l'administration d'application des lois et au plus important processus politique, ils ont perdu toute possibilité d'identifier un ennemi. Par conséquent, en dépit de la "frustration" économique générale, plus particulièrement au sein des couches sociales les plus défavorisées, les Afro-américains ont été incapables ces dernières années de se forger une identité forte. Cette constatation signifie-t-elle un déclin de l'idée de race dans la vie politique et économique américaine ? Oui et non. D'une part, nous pensons que les arguments développés ici permettent de dire qu'aujourd'hui, aux Etats-Unis, la notion de race est moins pertinente dans la perspective d'une action collective. Quoiqu'il en soit si nous observons malheureusement la détermination pour le bien être économique nous constatons que la race est un élément important et déterminant comme il y a 20 ou 30 ans. Pour répondre à notre question de pure forme, nous pouvons affirmer que les Etats-Unis ont certainement connu une expérience malheureuse dans le domaine des relations inter-raciales par rapport au reste du monde. Les Noirs qui ont été amenés en tant qu'esclaves, dans une nation jeune et composée d'immigrants, furent éparpillés ; on leur refusa une identité territoriale, élément crucial de la plupart des combats ethniques dans le monde entier. Plus important encore est l'existence d'un système basé sur la loi. La très forte référence au Bill of Rights a fourni à la nation son arme la plus puissante lors de troubles ethniques. De plus, l'évolution administrative depuis les années soixante a été absolument remarquable. De gros efforts ont été faits dans les domaines de la police et de la justice. On a, de même, par l'intermédiaire du L.E.A, favorisé dans les département de l'exécutif la sensibilisation aux problèmes raciaux. En bref, la nation a poursuivi une politique de justice procédurale au lieu d'entreprendre une politique de justice distributive. Avec le recul, on peut penser que la première s'est avérée plus efficace que la seconde car privilégiant le sentiment "d'impartialité". Les conclusions ci-dessus ne signifient certes pas pour autant que le pays a résolu ces problèmes raciaux. Loin de là. L'absence de conflit ouvert ne veut pas dire paix, ni le silence obstiné l'harmonie. Une telle concentration de misère économique sur un groupe ethnique ne manquera pas de créer des problèmes aux générations à venir, surtout lorsque que les dernières indications sur l'écart existant entre les Blancs et les Noirs font état d'une aggravation. Toutefois puisque le coeur du problème noir est devenu ou est en train de devenir un problème économique plus qu'un problème racial, il est probable que nous n'assistions pas à un soulèvement politique de masse à l'image de ce qu'avait appréhendé la Commission Kerner. Nous voyons et continuons à voir en revanche une escalade de la violence sociale et psychologique née de la frustration individuelle et du désespoir. L'on pourrait en fait se demander si les groupes constitués par les Noirs dans la perspective d'un trafic de drogue peuvent intégrer des idéologies politiques, ce qui pourrait par la suite les transformer en cellules d'organisations terroristes. Etant donnée l'hyper-ségrégation exercée sur les pauvres, cela pourrait devenir un problème chronique pour les classes sociales les plus basses et la classe des travailleurs où le faible niveau de violence ethnique pourrait influencer un avenir prévisible. Les Etats-Unis ont certainement quelque chose d'important à enseigner au reste du monde. Mais dans le même temps, tant que l'injustice économique persistera, elle pourra se manifester sous une forme aussi affreuse que dans certaines autres régions du monde. 
figure 1 : Comparaison du revenu moyen en dollars constant (1989) entre noir (courbe du bas) et blanc (courbe du haut). figure 2 : La pauvreté aux Etats-Unis de 1959 à 1988. Pourcentage de personnes vivant sous le seuil de pauvreté. (Source: Current Population Surveys) figure 3 : Pourcentage de noirs dans les emplois de bureau. figure 4: Pourcentage d'évolution relative dans la structure de classe des communautés noires.(Source : U. S. decennial Census)

figure 5 : Taux de croissance du revenu d'une famille afro-américaine.(distribution par groupe en fonction du niveau de revenu). Groupe 1 : moins de $10000 \$$ / Groupe 2 plus $10000 \$$, mais moins $25000 \$$ / Groupe 3 plus de $25000 \$$ mais moins de $35000 \$$ / Groupe 4 plus de $35000 \$$ mais moins de $75000 \$$ / Groupe 5 plus 75 000\$. Figure 6: Taux supérieur à l'indice 0,5 pour les officiers de police afro-américains.(Source: U. S. Decennial Census). Figure 7: Evolution des dépenses totales de la justice criminelle américaine (en millions de dollars - en dollar constant 1984). (Source : département de la Justice des Etats-Unis)

figure 8 : facteurs de motivation individuelle et environnement externe.

Pourcentage d'afro-américains sans revenu (par classe d'âge) entre 1969 et 1984. (source : Current Population Surveys)

classe d'âge 19691984 évolution en \%

$20-24162875$

$25-54816100$

$55-64224291$

tableau 1

\section{NOTES}

1. Kerner O., Report of the National Advisory Commission on Civil Disorders, 1968, Washington, D. C., U.S. Government Printing Office.

2....

3. Kerner O., op. cit., p. 1.

4. Kerner O., op. cit., p. 5.

5. National Research Council, A Common Destiny : Blacks and American Society, Washington D. C., National Academy Press. 1989, p. 6.

6. Cette chute des revenus n'était pas uniquement limitée aux noirs. Les blancs étaient aussi perdants, en terme de revenu réel, durant cette période, même si leurs pertes étaient considérablement moins importantes que celles des noirs.

7. Smith James P., Finis R. Welch, Closing the Gap, Forty Years of Economic Progress for Blacks, Santa Monica CA, Rand Corporation, 1986.

8. National Research Council, A Common Destiny : Blacks and American Society, Washington D. C., National Academy Press. 1989, p. 25. 
9. Massey Douglas S., Nancy A. Denton, "Hypersegregation in U.S. Metropolitan Areas : Black and Hispanic Segregation Along Five Dimensions", Demography, n² 26, p. 373 $391,1989$.

10. Olson (M.), The Logic of Collective Action, Cambridge, Harvard University Press, 1971.

11. Sen Amartya K., On Ethics and Economics, Oxford, Basil Blackwell, 1987.

12. Tullock (G.), "The Paradox of Revolution", Public Choice, $n^{\circ}$ 11, p. 89-99, 1971.

13. Cf. par exemple Gupta Dipak K., The Economics of Political Violence : The Effect of Political Instability on Economic Growth, New York, Praeger, 1990. Gupta Dipak K. and Harinder Singh, "Collective Rebellious Behavior : An Expected Utility Approach of Behavioral Motivations", Political Psychology, October 1992.

14. Pour renforcer ce point de vue, le professeur Amartya k. Sen remarque de façon poignante : "Pourquoi le fait de poursuivre son intérêt propre à l'exclusion de tout autre chose devrait-il être uniquement rationnel ?". Bien sûr, il n'est peut être pas complètement absurde de déclarer que la maximisation de l'intérêt personnel n'est pas irrationnel, du moins pas nécessairement, mais prétendre que tout ce qui ne relève pas de la maximalisation de l'intérêt doit être irrationnel semble tout autant invraisemblable" in On Ethics and Economics, Oxford, Basil Blackwell., p. 15. 15. Erikson Erik, Identity, Youth and Crisis, New York, W. W. Norton, 1968.

16. Tajfel (H.), "Aspects of Nationality and Ethnic Loyalty", Social Science Information, 1970, p. 113-144 ; Differentiation between Social Groups : Studies in Intragroup Relations. London : Academic Press, 1978.

17. Une partie croissante de la littérature en psychologie, en science politique, en économie, en sociologie, défend la considération de cet autre "moi". Pour un excellent recueil d'essais, voir Mansbridge Jane J. (ed.), Beyond Self Interest, Chicago, University of Chicago Press, 1990.

18. Pour une discussion sur ce qui est défini généralement comme revenu dans l'analyse politique, voir Lasswell H., Kaplan A., Power And Society : A Framework For Political Inquiry, New Haven, Yale University Press, 1950.

19. Bien que nous présentions ces deux biens utilitaires comme séparés, ils sont fortement interconnectés. Si la discrimination prend fin, ce sera un bien public pour ceux qui jusqu'à présent étaient victimes de cette discrimination, dans le sens où ce changement de condition sera bénéfique à tous ceux qui souffraient de cette discrimination. Elle créera en même temps pour ces gens des opportunités individuelles d'améliorer leurs propres sorts économiques. Ainsi la fin de l'apartheid en Afrique du sud peut signifier que les Noirs en général peuvent s'attendre à une meilleure situation économique. Les attentes d'un groupe relatives à l'obtention de biens privés peuvent bien sûr être différentes, elles reposent sur les capacités d'un individus. Ainsi la fin de la discrimination ouverte aux Etats-Unis a effectivement divisé la communauté noire en deux groupes : celui qui a pu tirer avantage du changement de situation grâce à son éducation, ces capacités, ces compétences, et grâce à d'autres facteurs d'opportunité ; celui qui mal préparé à ce changement a été abandonné à son propre sort et laissé pour compte.

20. Popkin (S.), The Rational Peasant, Berkeley, University of California Press, 1979 ; Axelrod (R.), "An Evolutionary Approach to Norms", American Political Science Review, $\mathrm{n}^{\circ} 80,1986$, p. 1095-1112.

21. Nous supposons que le coût de participation à une activité économique est nul, qu'il n'existe pas. 
22. Les exemples de forte croissance économique apaisant une nation récalcitrante, même soumis à un régime politique répressif, sont nombreux. La Corée du sud sous Park-Chung Hee ou le Chili sous Augusto Pinochet par exemple. Voir Johnson Chalmers, "The Democratization of South Korea : What Role Does Development Play ?", Paper presented at the Second Ilhae-Carnegie Conference on Democracy and Political Institutions, The Ilhae Institute, Soul, Korea, July 8-9, 1988.

23. Smelser Neal J., Theory of Collective Behavior, New York, Free Press of Glencoe, 1963.

24. Bien que l'on puisse s'attendre à une chute des actes politiques de défiance dans le cas d'une coercition gouvernementale rigide, il existe un débat important autour des actes de coercicion du gouvernement analysés soit comme une dissuasion soit comme une provocation "justifiant" une action ultérieure. Bien que Smelser, op. cit., Johnson, op. cit., prétendaient à l'existence d'une relation inverse entre les deux, Gurr soutenait avec Feierabend et d'autres, , avoir trouver la preuve d'une relation quadratique (Feierabend, I., R. Feierabend, B. Nesvold, "Social Change and Political Violence : Cross National Patterns" in Graham and Gurr (eds.), Violence in America : Historical and Comparative Perspectives, A Report to the National Commission on the Causes and Prevention of Violence, New York, Signet Books, 1969. Pour des développements plus récents sur cette relation complexe voir Lichbach I. L., "Deterrence or Escalation? The Puzzle of Aggregate Studies of Repression and Dissent", Journal of Conflict Resolution, n 31, 1987, p. 266-297 ; Gupta Dipak K., Harinder Singh, Tom Sprague, "Government Coercion : Deterrence or Provocation? An Empirical Rejoinder", School of Public Administration and Urban Studies, San Diego State University., 991.

25. Pour une mesure empirique de l'idéologie de groupe voir Gupta Dipak K.,The Economics of Political Violence : The Effect of Political Instability on Economic Growth, New York, Praeger, 1990 ; Gupta Dipak K., Harinder Singh, "Collective Rebellious Behavior : An Expected Utility Approach of Behavioral Motivations", Political Psychology, October 1992 ; Gupta Dipak K., Richard Hofstetter, "Collective Choice and Group Identity", School of Public Administration and Urban Studies, San Diego State University, 1991.

26. Frederik Douglass, mentionne par exemple dans son autobiographie : "Le lecteur ne doit pas s'attendre à ce que je parle beaucoup de ma famille. Ma première expérience de la vie, autant que je puisse m'en souvenir maintenant (...) commençait dans la famille de ma grand-mère et mon grand-père. (...) la pratique de séparation des enfants de leurs mères, pour les louer, à des distance trop grandes pour permettre une rencontre, pendant de longues périodes, fut le trait caractéristique de la cruauté et de la barbarie du système de l'esclavage. Il n'avait pas d'intérêt à reconnaître ou à préserver les liens qui unissaient les familles ou leurs maisons. (Life and Times of Frederick Douglass, New York, Macmillan first published in 1892, 1962, p. 27). 27. Wilson William J., The Declining Significance of Race : Blacks and Changing American Institutions. Chicago, The University of Chicago Press, 1978.

28. Wilson William J., op. cit.

29. Pour une excellente discussion autour de l'explication par le choix rationnel du mouvement des droits civiques voir Dennis Chong, Collective Action and the Civil Rights Movement, Chicago, University of Chicago Press, 1991.

30. Tullock G., "The Paradox of Revolution", Public Choice, $\mathrm{n}^{\circ}$ 11, p. 89 -99, 1971. 31. Banfield E. C., The Un-Heavenly City : The Nature and the Future of Our Urban Crisis, Boston, Little, Brown, 1968. 
32. Pour une discussion autour des participants aux émeutes plus orientés idéologiquement voir Caplan N., "The New Ghetto Man : A Review of Recent Empirical Findings", Journal of Social Issues, $n^{\circ} 26,1970$, p. 59-73 ; Feagin J. R., H. Hahn, Ghetto Revolts : The Politics of Violence in America, New York, Macmillan \& Co., 1973 ; Fogelson, R., Violence as Protest, Garden City, N. Y., Doubleday, 1971 ; Geshwender J. A., "Civil Rights Protests and Riots : A Disappearing Distinction", Social Science Quarterly, $\mathrm{n}^{\circ}$ 49, 1968, p.479-493 ; Lupsha P., "Explanation of Political Violence : Some Psychological Theories Versus Indignation", Politics and Society, $n^{\circ}$ 2, 1969, p. 80-104 ; Sears D. O., J. B. McConahey, The Politics of Violence : The New Urban Black and the Watts Riot, Boston, Houghton Mifflin, 1973.

33. Les observateurs étrangers ont tendance à surestimer les capacités d'action collective des groupes émeutiers de Los Angeles. Pour une discussion voir Cultures et Conflits $n^{\circ} 5$ sur "Les émeutes urbaines" et Cultures et Conflits $n{ }^{\circ} 6$ sur "Les violences urbaines".

34. Le chiffre correspondant pour les Blancs est $40,2 \%$. Il est intéressant de noter cependant que parmi les Blancs le groupe des plus haut revenus a connu un gain proportionellement similaire durant la même période de temps. (183\% pour les Blancs, $189 \%$ pour les Noirs) avec les nombreuses augmentations qui vont suivre durant les années 80.

35. Massey Douglas S., Nancy A. Denton, "Hypersegregation in U.S. Metropolitan Areas : Black and Hispanic Segregation Along Five Dimensions", Demography, n² 26, p. 373 391, 1989.

36. Pour une discussion extrêmement intéressante sur la dimension psychologique du besoin d'un « Eux » identifiable qui encourage l'esprit collectif du « Nous », voir Volkan V. D., The Need to Have Enemies \& Allies : From Clinical Practice to International Relations, Dunmore, Penn., Jason Aronson, 1988. Volkan intègre la psycho-analyse de l'individu avec le comportement national et international.

37. « Losing Ground », Newsweek, 6 avril 1992, p. 21-22.

38. ...

39. op. cit., p. 165.

40. Lewis William G., "Toward Representative Bureaucracy : An Assessment of Black Representation in Police Bureaucracies", Preliminary results of Ph. D. dissertation research. Public Administration Review, $n^{\circ}$ 49, 1987, p. 257-268.

41. L'index de l'Equal Employement Opportunité est construit sur une base de parité raciale.

42. Alors que l'évidence suggère que les communautés noires dans les secteurs des centres villes peuvent considérer les forces de police comme leur ennemi, sans identification ethnique claire, la notion d'ennemi demeure vague.

43. Knowles Louis L., Kenneth Prewitt, "Racism in the Administration of Justice." in C. E. Rasons and J. L. Kuykendall (eds.), Race, Crime, and Justice. Pacific Palisades, Calif., Goodyear, 1972, pp. 13-27.

44. Marx Gary T., "External Efforts to Damage or Facilitate Social Movements : Some Patterns, Explanations, Outcomes, and Complications", in J. Wood and M. Jackson (eds), Social Movements : Development, Participation, and Dynamics, Belmont, Calif., Wadsworth Publishing, 1982, pp. 181-200.

45. Marx Gary T., op. cit., p. 183. 


\section{RÉSUMÉS}

Alors que les conflits raciaux, ethniques, dominent continuellement les actualités, les Etats-Unis goûtent un calme relatif. Peu de personnes auraient parié, il y a encore peu de temps, sur cette relative tranquilité. En effet, il y a à peine un quart de siècle les Etats-Unis étaient confrontés à une vague d'émeutes urbaines sans précédent et aux mouvements pour les droits civiques extrêmement déterminés. Le rapport de la Commission Kerner, mise en place à la suite de ces émeutes et chargée d'en étudier les causes, avait prédit à l'époque l'imminence d'un affrontement généralisé encore plus violent, si des efforts importants n'étaient pas entrepris pour combler le gouffre économique séparant les communautés noires des communautés blanches. Cependant des études récentes indiquent, qu'en moyenne, les conditions socioéconomiques des communautés noires se sont depuis, encore dégradées, sans pourtant déboucher sur une multiplication des violences de la part des communautés noires, bien au contraire. Le calme relatif qui caractérise donc les relations inter-raciales aux Etats-unis n'est donc pas à attribuer à la dégradation des "conditions de vie" des communautés noires mais peutêtre à la difficile construction d'une identité propre.

\section{INDEX}

Index géographique : Etats-Unis

Mots-clés : racisme, émeutes 